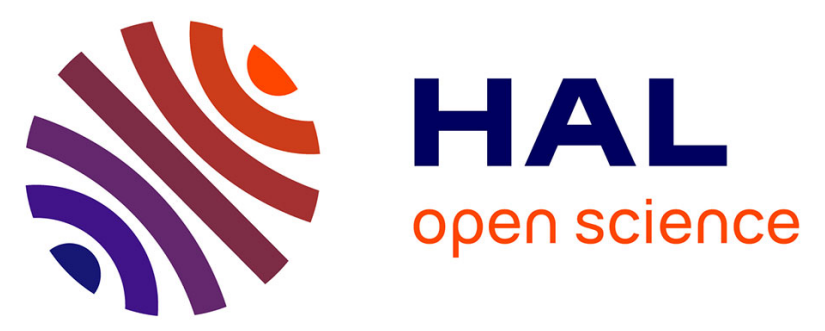

\title{
Conventional Matrices Loaded Onto a Graphene Layer Enhances MALDI-TOF/TOF Signal: Its Application to Improve Detection of Phosphorylated Peptides
}

Carlos Rodriguez, Javier Palacios, Ignacio Fajardo, José Luis Urdiales, Xavier Le Guével, Jose Lozano, Francisca Sánchez-Jiménez

\section{- To cite this version:}

Carlos Rodriguez, Javier Palacios, Ignacio Fajardo, José Luis Urdiales, Xavier Le Guével, et al.. Conventional Matrices Loaded Onto a Graphene Layer Enhances MALDI-TOF/TOF Signal: Its Application to Improve Detection of Phosphorylated Peptides. Journal of The American Society for Mass Spectrometry, 2016, 27 (2), pp.366-369. 10.1007/s13361-015-1299-3 . hal-02338004

\section{HAL Id: hal-02338004 https://hal.science/hal-02338004}

Submitted on 29 Oct 2019

HAL is a multi-disciplinary open access archive for the deposit and dissemination of scientific research documents, whether they are published or not. The documents may come from teaching and research institutions in France or abroad, or from public or private research centers.
L'archive ouverte pluridisciplinaire HAL, est destinée au dépôt et à la diffusion de documents scientifiques de niveau recherche, publiés ou non, émanant des établissements d'enseignement et de recherche français ou étrangers, des laboratoires publics ou privés. 


\section{Conventional Matrices Loaded Onto a Graphene Layer Enhances MALDI-TOF/TOF Signal: Its Application to Improve Detection of Phosphorylated Peptides}

Carlos Rodriguez, Javier Palacios, Ignacio Fajardo, José Urdiales, Xavier Le Guével, Jose Lozano, Francisca Sánchez-Jiménez, José Luis Urdiales

\section{To cite this version:}

Carlos Rodriguez, Javier Palacios, Ignacio Fajardo, José Urdiales, Xavier Le Guével, et al.. Conventional Matrices Loaded Onto a Graphene Layer Enhances MALDI-TOF/TOF Signal: Its Application to Improve Detection of Phosphorylated Peptides. Journal of The American Society for Mass Spectrometry, Springer Verlag (Germany), 2016, 27 (2), pp.366-369. 10.1007/s13361-015-1299-3 . hal-02338004

\section{HAL Id: hal-02338004 \\ https://hal.archives-ouvertes.fr/hal-02338004}

Submitted on 29 Oct 2019

HAL is a multi-disciplinary open access archive for the deposit and dissemination of scientific research documents, whether they are published or not. The documents may come from teaching and research institutions in France or abroad, or from public or private research centers.
L'archive ouverte pluridisciplinaire HAL, est destinée au dépôt et à la diffusion de documents scientifiques de niveau recherche, publiés ou non, émanant des établissements d'enseignement et de recherche français ou étrangers, des laboratoires publics ou privés. 


\title{
Conventional Matrices Loaded Onto a Graphene Layer Enhances MALDI-TOF/TOF Signal: Its Application to Improve Detection of Phosphorylated Peptides
}

\author{
Carlos E. Rodríguez, ${ }^{1}$ Javier Palacios, ${ }^{1}$ Ignacio Fajardo, ${ }^{2,3}$ José Luis Urdiales, ${ }^{2,3}$ \\ Xavier Le Guével, ${ }^{5}$ José Lozano, ${ }^{2,4}$ Francisca Sánchez-Jiménez ${ }^{2,3}$ \\ ${ }^{1}$ Proteomics Unit of the Supercomputing and Bioinnovation Center of the University of Málaga, Parque Tecnológico de Andalucía, \\ Málaga, Spain \\ ${ }^{2}$ Department of Molecular Biology and Biochemistry, University of Malaga, Málaga, Spain \\ ${ }^{3}$ Unit 741 of Centro de Investigación en Red en Enfermedades Raras (CIBERER-ISCIII), Malaga, Spain \\ ${ }^{4}$ Laboratorio de Oncología Molecular, Servicio de Oncología Médica, Instituto de Biomedicina de Málaga (IBIMA), Hospital \\ Universitario Virgen de la Victoria, Málaga, Spain \\ ${ }^{5}$ Therapeutic Nanosystem, Andalusian Center for Nanomedicine and Biotechnology (BIONAND), Parque Tecnológico de \\ Andalucía, Campanillas, Málaga, Spain
}

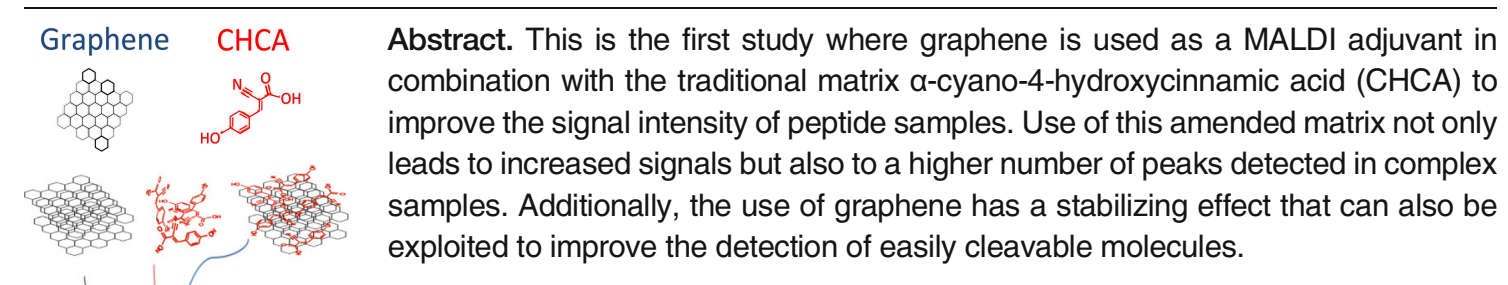

Keywords: Graphene, MALDI-TOF/TOF, Phosphorylated peptides

Received: 26 July 2015/Revised: 22 October 2015/Accepted: 24 October 2015

\section{Introduction}

T $\mathrm{n}$ the last few years, the use of graphene as a new MALDI 1 matrix for the analysis of low molecular weight molecules has been extensively described [1-6]. The main advantages of this matrix are related to (1) the absence of interfering peaks in the spectra at low mass range, usually visible when using traditional matrices [7], and (2) an attenuation of analyte fragmentation [8], which could reduce the complexity of the spectra at higher signal intensities.

Graphene has ultrafast electron mobility with an efficient electron-phonon coupling, as well as frequency-independent optical absorption properties and a high thermal conductivity [9].

Electronic supplementary material The online version of this article (doi:10. 1007/s13361-015-1299-3) contains supplementary material, which is available to authorized users

Correspondence to: Carlos E. Rodríguez; e-mail: acrge1@gmail.com Published online: 30 November 2015
Our hypothesis is that the good adsorption of the peptides and other molecules in the planar structure of the graphene together with its exceptional optical and electronic properties facilitates the ionization of the analytes. More ionization associated with a decreased vibrational energy during the laser ablation would lead to an improvement of the signal, especially of those species that fragment easily. Following this hypothesis, we set as the main objective of this work testing the putative advantages of using graphene in combination with $\alpha$-cyano-4-hydroxycinnamic acid (CHCA) as a binary matrix system to combine the excellent electrical, optical, thermal, and adsorbent properties of graphene with the intense resonant light absorbtion of CHCA to enhance the signal in MALDI-TOF mass spectrometry.

The proper detection of molecules that are easily fragmented by MALDI-TOF mass spectrometry is an underestimated problem. For example, although the phosphate group is easily ionizable, the detection of phosphopeptides can be frequenly missed owing to the lability of the phosphoester bond, which shows a 
high fragmentation rate. Here, we have tested the analysis of phosphopeptides with the combination of graphene/CHCA and have found a significant improvement over the use of CHCA alone.

\section{Experimental}

\section{Graphene Preparation}

Graphene (reduced graphene oxide [10]) was acquired from GRAPHENEA (San Sebastián, SPAIN). CHCA was prepared as a saturated solution in 50\% acetonitrile, $0.1 \%$ TFA, and sinapinic acid as a saturated solution in $30 \%$ acetonitrile $0.1 \%$ TFA. One mg of the graphene powder was resuspended in $0.5 \mathrm{~mL}$ of $50 \%$ ethanol using a low power bath sonicator (Selecta Ultrasons, JP Selecta, Barcelona, Spain) for $3 \mathrm{~min}$ in a glass vial.

\section{Mass Spectrometry}

A 4700 Proteomics Analyzer Mass Spectrometer (AB SCIEX, Framingham, MA. USA) was used for the analyses. All the details of the conditions employed are described in the Supporting Information.

\section{Results and Discussion}

\section{Graphene Enhances MALDI-TOF MS Signal Intensity}

Graphene has already been successfully employed as a MALDITOF matrix for the analyses of small molecules $(<450 \mathrm{Da})[2,7$, $8]$ but fails when used to detect larger molecules. A simple addition of $1 \mu \mathrm{L}$ of the matrix CHCA onto the same spots where the mixture of graphene-analyte was loaded in the MALDI plate not only allowed the recovery of the signal for larger molecules but also caused an improvement of signal intensities, signal-tonoise ratios, and mass resolving power.

This improvement showed a strong dependence on the graphene and CHCA loading method (see Supporting Information for details), and the best results were obtained by adding the graphene first, allowing it to dry, and subsequently loading the sample and the matrix (previously mixed in a tube with vortexing) onto the graphene layer. The combination of graphene/CHCA not only increased the signal of the 4700 proteomics analyzer calibration mixture (calmix) compared with the measurement accomplished with only $\mathrm{CHCA}$ but also reduced the laser intensity needed to ionize the sample. For laser intensities below 2700 arbitrary units, no signal was observed when using only $\mathrm{CHCA}$ as the matrix, while we were able to detect the signal with graphene/CHCA at a laser intensity of 2500 .

Different ratios of graphene:CHCA as matrices were subsequently tested using calmix as the sample. Under the experimental conditions described in Figure 1a, the best result was obtained by loading $1 \mu \mathrm{L}$ of graphene and $1 \mu \mathrm{L}$ of CHCA.

Sinapinic acid with graphene was also evaluated as a binary matrix system with the same loading protocol established for CHCA. In this case, the sample used was the calibration mixture 3 of the Sequazyme Peptide Mass Standard Kit (AB SCIEX).

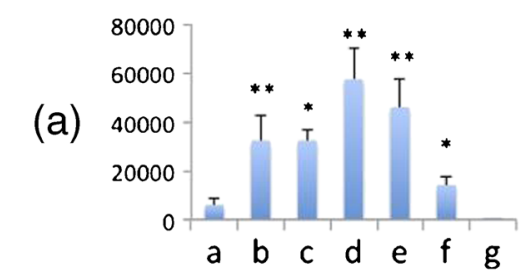

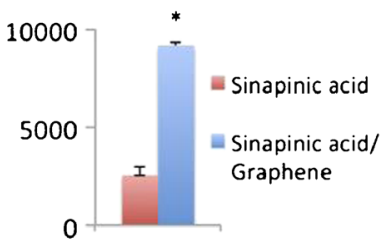

(b)

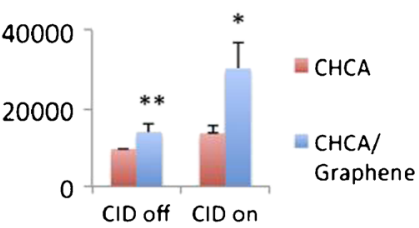

(c)
Figure 1. Enhancement of MALDI-TOF/TOF MS signal by graphene in combination with $\mathrm{CHCA}$ matrix. (a) Calmix spectra were obtained with five different ratios of graphene/CHCA in reflector positive-ion mode. One $\mu \mathrm{L}$ of calmix was employed and the same laser intensity (2900) in all tested conditions. Graphene/CHCA ratios were $(\mu \mathrm{L}: \mu \mathrm{L}): \mathbf{a}, 0: 1 ; \mathbf{b}, 0.25: 1 ; \mathbf{c}, 0.5: 1$; d, $1: 1 ; \mathbf{e}, 1: 0.5 ; \mathbf{f}, 1: 0.25 ; \mathbf{g}, 1: 0$. (b) Comparison of the signal intensities obtained with sinapinic acid and graphene/sinapinic acid of linear low mass positive-ion mode spectra. (c) Comparison of the signal intensities obtained with $\mathrm{CHCA}$ and graphene/ CHCA of product-ion spectra carried out in CID-off and CID-on modes. Results are mean \pm SEM of three independent experiments. ${ }^{*} P<0.05$; ${ }^{\star \star} P<0.01$ compared with single matrix (nonbinary) by Student's paired sample $t$-test (two-tailed)

Figure $1 \mathrm{~b}$ shows that the signal was enhanced approximately 2fold compared with the response with the sinapinic acid alone.

\section{Utility of the Binary Matrix for Complex Samples}

A digestion of a complete protein extract derived from human leukocytes was studied to evaluate the usefulness of the graphene/ CHCA combination to analyze complex samples (Figure 2). With the binary matrix, we detected a higher number of peaks than when using only CHCA at any laser intensity. Comparison of the best spectra for both conditions (CHCA alone and graphene/ CHCA combination) showed an increase in the number of peaks detected. This peak detection enhancement was observed mainly in the high mass range, and the mass resolving power of the spectra was significantly improved at this range.

We have observed that the loading conditions when using calmix as the sample are not always suitable for complex samples or for samples with interfering substances. In these cases, it is recommended to perform an initial condition screening to optimize the results. Nevertheless, to have a clearer presentation of the data, the results shown have always been carried out using the protocol D, described in the paragraph 'Loading of matrices and samples' of the Supporting Information.

\section{PSD and CID Fragmentation Comparison}

As shown in Figure 1c, the signal improvement with the use of graphene/CHCA was higher when CID-on mode was employed $(53 \% \pm 12$ CI $95 \%)$ compared with with PSD MS/MS mode 


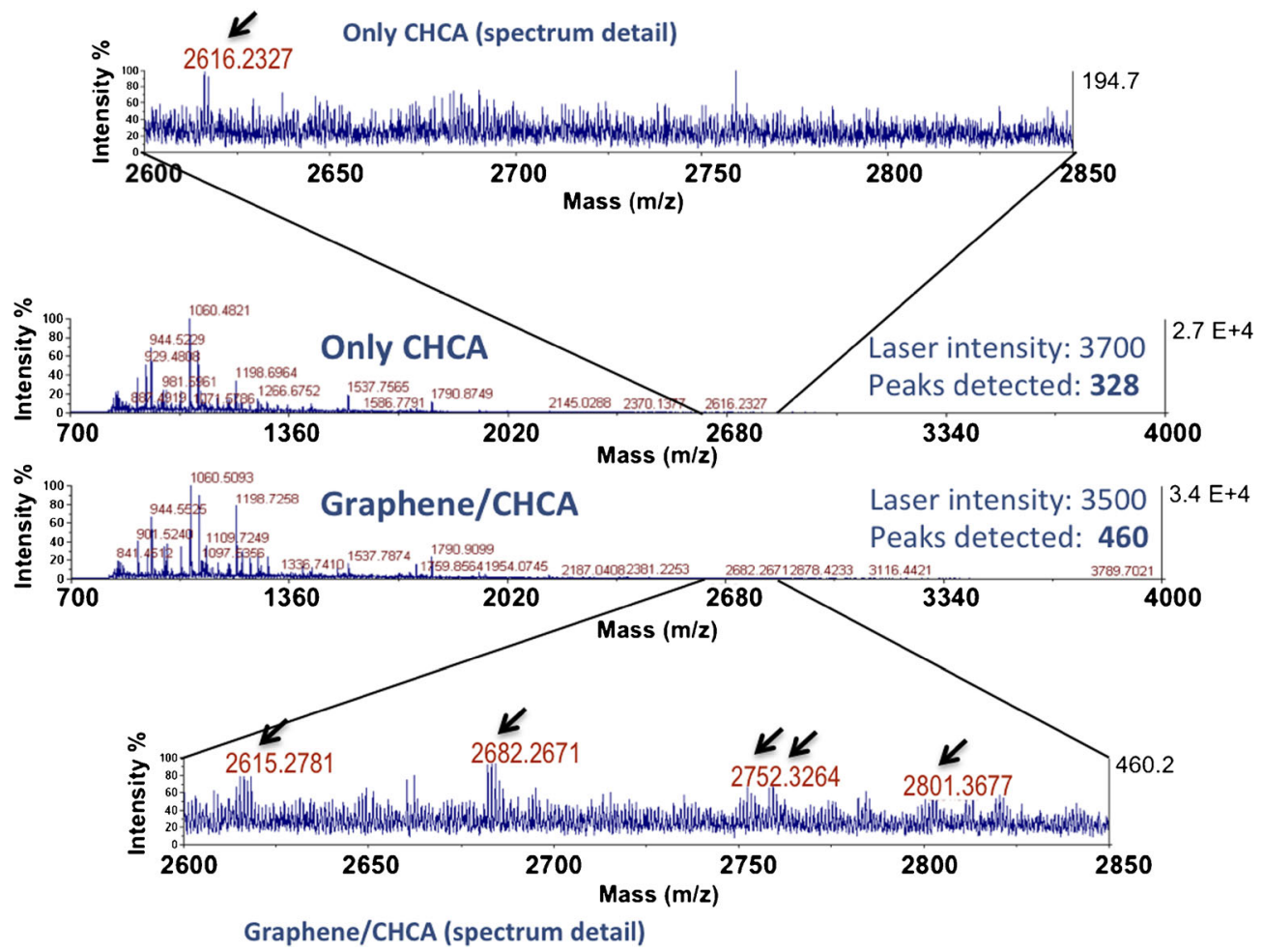

Figure 2. MALDI-TOF MS signal obtained from a complex sample without and with the use of graphene in combination with CHCA matrix. A protein extract of isolated leukocytes was digested with trypsin, and the spectra were obtained in reflector positive ion mode with CHCA or graphene/CHCA as matrices. Spectrum details in the 2600-2850 m/z range showed an increased number of peaks detected with the binary matrix system. Peaks detection conditions were: min S/N: 10, local noise window width: 250 , min peak width at full width half mass (bins): 2.9 , cluster area S/N optimization: on, S/N threshold: 15

$(30 \% \pm 12$ CI 95\%). This also corroborates the idea that the graphene facilitates the analyte ionization that can be achieved with lower energy collisions between the molecules of matrix and analyte. In this condition, the molecules fly with lower vibrational energy toward the detector and higher signal is obtained with the CID on mode when it is compared with the spontaneous fragmentation of the PSD mode.

\section{Improved Detection of Phosphorylated Peptides by Graphene/CHCA Matrix}

Bearing in mind the attenuating fragmentation effect of graphene, we decided to study samples containing easily fragmented post- translational modifications, such as phosphorylated peptides. In Figure 3, an example is shown where we could not detect an expected phosphorylation with the use of CHCA alone as a matrix, which was readily detectable upon the use of graphene/ CHCA. The peak at $\mathrm{m} / \mathrm{z} 1758.9$ was apparently absent when using only CHCA as a matrix, but was clearly observed by using the combination of graphene/CHCA as a matrix. The sequence of the phosphopeptide in the peak of $\mathrm{m} / \mathrm{z}$ 1758.9: ALHSFITPPTT*PQLR (where $T^{*}$ is the phosphorylated residue) was confirmed by MS/MS (see Supporting Information).

Serial dilutions of a solution of digested casein were also analyzed. The ions of $m / z 1660.7$ and 1951.8 in Figure 2S (Supporting Information) correspond to the following sequences

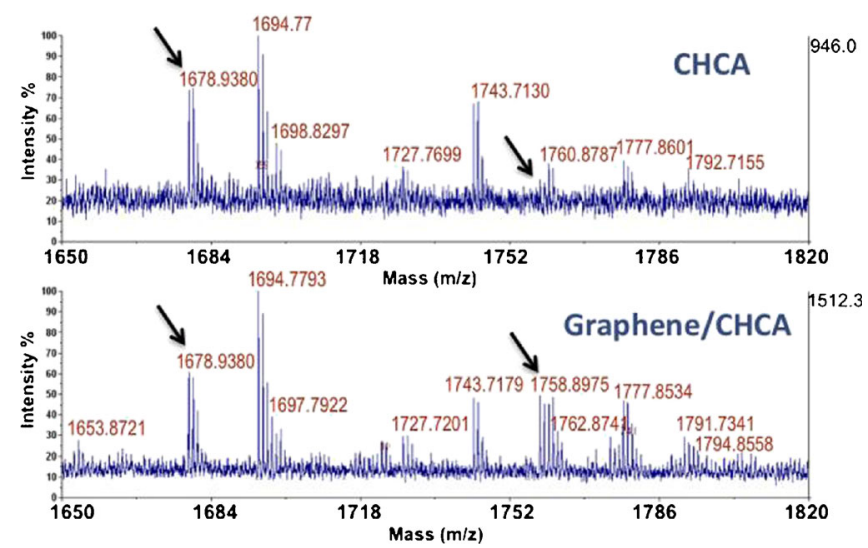

Figure 3. Detail of the mass spectra obtained for phosphorylated KSR 1 with or without the use of graphene in the matrix 
of the widely studied tryptic peptides phosphorylated with casein: VPQLEIVPNS*AEER (0 missed cleavage) and YKVPQLEIVPNS*AEER (1 missed cleavage). Their sequences were confirmed by MS/MS analysis. Both peaks were observed with both matrices without any dilution. However, the peptide ion of $\mathrm{m} / \mathrm{z} 1951.8$ was lost when only CHCA was used with a sample dilution of 1:10, while it was still clearly seen with graphene/CHCA. It is important to note that this effect was observed when the samples were desalted with ZipTips but not when the samples were not previously desalted.

The signal enhancement of different peptides obtained using graphene as a binary matrix system with CHCA can be explained on the basis of several effects. On the one hand, the exceptional optical and thermal properties of the graphene give rise to a high ionization yield where the analyte molecules get less vibrational excitation, thereby reducing the fragmentation rate during their flight to the detector. On the other hand, it cannot be ruled out that the main effect is promoted by interaction with the delocalized electron charge state of the graphene surface. The fact that the graphene alone can be successfully used as a matrix for small molecules is an argument that also supports this idea.

Moreover, the fact that the product-ion signal intensity was significantly more increased in the CID than in the PSD fragmentation mode supports the idea that the graphene is not only able to enhance the ionization rate but also lead to a stabilization effect that increases the number of ions reaching the detector. These findings are in agreement with those of Liu et al. [2] who first suggested the attenuating effect of graphene on the spontaneous fragmentation of molecules flying towards the detector. This attenuation of the fragmentation rate could explain, at least partially, the enhancement of the signal obtained.

Experiments carried out with phosphorylated peptides also support this hypothesis. We showed that their detection is enhanced by the use of graphene/CHCA, and the most plausible explanation could come from the stabilizing effect of the graphene.

\section{Conclusions}

The use of graphene as a MALDI matrix was first published in 2010 [8]. Since then, other articles have described the use of graphene as a matrix [1, 3-5], and the present work is the first study where graphene is used as a MALDI adjuvant improving ionization rate with a stabilizing effect that reduces the rate of fragmentation and gives rise to a better detection of phosphorylated peptides and a higher number of peaks detected in the analysis of complex samples. The enhancement of the signal with the combination of graphene/CHCA is especially useful in the case of complex samples where more peaks are detected, mainly in the high mass range of the spectra.

Further studies to test graphene-coated MALDI plates or even plates completely made of this substance can be of great interest. Under these conditions, the undesirable aggregation of the graphene might be avoided [2], which may significantly reduce the desorption/ionization efficiency.
In spite of exhibiting a frequency-independent light absorption over a broad spectral region in the visible and infrared, graphene has a characteristic absorption band in the range of 250-290 nm (Figure 1S of Supporting Information). There are very few published papers in which graphene is used as matrix for MALDITOF and all of them employed mass spectrometers equipped with a laser emitting at $337 \mathrm{~nm}$ (pulsed nitrogen laser) [8] or $355 \mathrm{~nm}$ (Nd:YAG laser) $[3,8]$; both wavelengths are far from the maximum absorption wavelength of graphene $(270 \mathrm{~nm})$ [9]. In these cases, the processes involved in the ionization of the molecules must be related to surface electronic charge events rather than a resonant absorption of light, as is the case with traditional matrices used in MALDI-TOF mass spectrometers. In this sense, it would be of great interest to design experiments using a laser emitting at this wavelength interval $(250-290 \mathrm{~nm})$ to test the performance of this novel matrix with a resonant light absorption.

\section{Acknowledgments}

The SCBI Proteomics Unit of the University of Málaga (Parque Tecnológico de Andalucía) enabled the present work to be carried out. It was supported by grants SAF2011-26518 (MINECO, Spain), SAF2010-20203 (MINECO Spain), and CVI-06585 (PAIDI, Andalusia). CIBERER is an initiative of Institute de Salud Carlos III. The authors thankfully acknowledge the expertise, understanding, and assistance of the Nanotech Unit of the SCBI, for sharing support and encouragement.

\section{References}

1. Shi, C., Meng, J., Deng, C.: Enrichment and detection of small molecules using magnetic graphene as an adsorbent and a novel matrix of MALDITOF-MS. Chem. Commun. (Camb). 48, 2418-2420 (2012)

2. Liu, Y., Liu, J., Deng, C., Zhang, X.: Graphene and graphene oxide: two ideal choices for the enrichment and ionization of long-chain fatty acids free from matrix-assisted laser desorption/ionization matrix interference. Rapid Commun. Mass Spectrom. 25, 3223-3234 (2011)

3. Zhang, J., Dong, X., Cheng, J., Li, J., Wang, Y.: Efficient analysis of nonpolar environmental contaminants by MALDI-TOF MS with graphene as matrix. J. Am. Soc. Mass Spectrom. 22, 1294-1298 (2011)

4. Liu, Y., Liu, J., Yin, P., Gao, M., Deng, C., Zhang, X.: High throughput identification of components from traditional Chinese medicine herbs by utilizing graphene or graphene oxide as MALDI-TOF-MS matrix. J. Mass Spectrom. 46, 804-815 (2011)

5. Zhou, X., Wei, Y., He, Q., Boey, F., Zhang, Q., Zhang, H.: Reduced graphene oxide films used as Matrix of MALDI-TOF-MS for detection of octachlorodibenzo-p-dioxin. Chem. Commun. (Camb). 46, 6974-6976 (2010)

6. Wang, J., Liu, Q., Gao, Y., Wang, Y., Guo, L., Jiang, G.: High-throughput and rapid screening of low-mass hazardous compounds in complex samples. Anal. Chem. 87, 6931-6936 (2015)

7. Wan, D., Gao, M., Wang, Y., Zhang, P., Zhang, X.: A rapid and simple separation and direct detection of glutathione by gold nanoparticles and graphene-based MALDI-TOF-MS. J. Sep. Sci. 36, 629-635 (2013)

8. Dong, X., Cheng, J., Li, J., Wang, Y.: Graphene as a novel matrix for the analysis of small molecules by MALDI-TOF MS. Anal. Chem. 82, 6208-6214 (2010)

9. Liu, Q., Cheng, M., Jiang, G.: Mildly oxidized graphene: facile synthesis, characterization, and application as a matrix in MALDI mass spectrometry. Chemistry 19, 5561-5565 (2013)

10. Park, S., An, J., Potts, J.R., Velamakanni, A., Murali, S., Ruoff, R.S.: Hydrazinereduction of graphite- and graphene oxide. Carbon 49, 3019-3023 (2011) 